PROCEEDINGS OF THE

AMERICAN MATHEMATICAL SOCIETY

Volume 51, Number 1, August 1975

\title{
ABSOLUTELY CLOSED MAPS
}

\section{LOUIS FRIEDLER}

ABSTRACT. An example is given of a continuous function $f: X \rightarrow Y$ which is closed, has point inverses $H$-closed, but which can be extended to a continuous function $F: Z \rightarrow Y$ for some $Z$ which has $X$ as a proper dense subset. A partial characterization of nonextendable functions is given in terms similar to Bourbaki's theorem that perfect maps $f: X \rightarrow Y$ are those for which $f \times i_{Z}: X \times Z \rightarrow Y \times Z$ is a closed map for all spaces $Z$.

A map $f: X \rightarrow Y$ is called absolutely closed if there does not exist an extension of $f$ to $F: X^{*} \rightarrow Y$ where $X^{*}$ is any space with $X$ as a proper dense subset. These maps seem interesting in terms of extensions of functions and in their relation to $H$-closed spaces. Absolutely closed maps were first introduced by Blaszczyk and Mioduszewski [1] and characterizations have been given by Viglino [6] and Dickman [3].

All spaces are assumed Hausdorff and all maps continuous. A countable ultrafilter is one with a countable base. A subset $A$ of $X$ is called (countably) far from the remainder (c.f.f.r. or f.f.r. respectively) iff for each free open (countable) ultrafilter $\mathcal{U}$ on $X$, there is some $U \in \mathcal{U}$ for which $\bar{U} \cap A$ $=\varnothing$. A closed set is regular closed iff it is the closure of its interior. A map is regular closed iff the image of every regular closed set is closed.

Theorem 1 (Dickman [3]). A map $f: X \rightarrow Y$ is absolutely closed iff

(1) $f^{-1}(y)$ is f.f.r. for all $y \in Y$ and

(2) $f$ is regular closed.

Viglino [6] has asked whether every closed map with point inverses $H$ closed is absolutely closed. In view of Theorem 1, Dickman [3] pointed out that this question may be rephrased: if point inverses of a closed map are $H$-closed, are they also f.f.r.? The following example gives a negative answer to this question. The space used is essentially the one described in [1, p. 48].

Example. Let $X=\left([-1,1] \times N^{+}\right) \cup\{p\}$, where $N^{+}$is the set of positive integers and $p$ is an additional point whose neighborhoods are of the form

Received by the editors May 9, 1974.

AMS (MOS) subject classifications (1970). Primary 54C10, 54D25.

Key words and phrases. $H$-closed, absolutely closed map, regular closed map. 
$V_{n}=\{(x, i) \mid x>0, i \geq n\} \cup\{p\}$. Consider the subset $A$ of $X$ consisting of $\left([0,1] \times N^{+}\right) \cup\{p\} . X$ is clearly Hausdorff and $A$ is $H$-closed but $A$ is not f.f.r. Construct an ultrafilter $\mathcal{O}$ on $X$ as follows: let $\mathcal{U}=\mathcal{U}_{1} \cup \mathcal{U}_{2}$ where the sets in $\mathcal{U}_{1}$ are $U_{n}=\{(x, i) \mid x<0, i \geq n\}$ and the sets in $\mathcal{U}_{2}$ are of the form $U_{t_{1} t_{2} \ldots}=\left\{(x, n) \mid t_{n}<x<0,-1 \leq t_{n}<0\right\}$. Then $U$ is contained in some free ultrafilter $\mathcal{O}$. We claim that $\bar{V} \cap A \neq \varnothing$ for all $V \in \mathcal{O}$. If there were some $V_{0} \in \mathcal{C}$ for which $\bar{V}_{0} \cap A=\varnothing, \bar{V}_{0} \cap\left\{(0, n) \mid n \in N^{+}\right\}=\varnothing$. It follows that for each $n$ there exist $t_{n}$ and $s_{n}$ with $-1 \leq t_{n}<0,0<s_{n} \leq 1$ and $V_{0}$ disjoint from the interval $\left(t_{n}, s_{n}\right)$. But then $V_{0} \cap U_{t_{1} t_{2}} \ldots=\varnothing$, which is impossible. Thus $\bar{V} \cap A \neq \varnothing$ for all $V \in \mathcal{O}$ and $A$ is not f.f.r.

Identify $A$ to a point $y_{0}$ and let $f$ be the natural map and $Y$ the quotient space. Then $Y$ is Hausdorff, $f$ is closed, $f^{-1}(y)$ is $H$-closed for all $y \in Y$ but $f^{-1}\left(y_{0}\right)=A$ is not f.f.r. so that $f$ is not absolutely closed.

Remarks. It is well known [2] that a map $f: X \rightarrow Y$ is perfect iff $f \times i_{Z}: X \times Z \rightarrow Y \times Z$ is a closed map for all spaces $Z$, where $i_{Z}$ is the identity map on $Z$. In the special case that $Y$ is a point we obtain as a corollary Scarborough's result [4] that a space $X$ is compact iff the projection $\pi_{Z}: X \times Z \rightarrow Z$ is closed for all spaces $Z$. Our interest here is to examine to what extent similar results hold for absolutely closed functions. That is, what theorem gives as a corollary Scarborough's result [4] that a space $X$ is $H \cdot c$ closed iff the projection $\pi_{Z}: X \times Z \rightarrow Z$ is regular closed for all spaces $Z$ ? We conjecture that $f: X \rightarrow Y$ is absolutely closed iff $f \times i_{Z}$ : $X \times Z \rightarrow Y \times Z$ is regular closed for all spaces $Z$. The necessity is proved in Theorem 3 but for the sufficiency we only have the partial result of Theorem 2. Notice that Theorem 2 implies that for the function of the Example, there is a space $Z$ with $f \times i_{Z}: X \times Z \rightarrow Y \times Z$ not regular closed.

Definition. A map $f: X \rightarrow Y$ is countably absolutely closed if there does not exist an extension of $f$ to $F: X^{*} \rightarrow Y$ where $X^{*}$ is any space with $X$ as a proper dense subset such that all points of $X^{*}-X$ have a countable neighborhood base.

The proofs of the following lemmas follow the techniques of Theorem 1 above and Theorem 1.2 of [6] and are omitted. Lemma 1 also holds for arbitrary countable filter-bases.

Lemma 1. $f: X \rightarrow Y$ is countably absolutely closed iff no free countable ultrafilter $\mathcal{U}$ on $X$ has $f(\mathcal{U})$ convergent.

Lemma 2. If $f: X \rightarrow Y$ and

(1) $f$ is regular closed and 
(2) $f^{-1}(y)$ is c.f.f.r. for all $y \in Y$, then $f$ is countably absolutely closed. If $f$ is countably absolutely closed and $Y$ is first countable then (1) and (2) hold.

Theorem 2. Let $f: X \rightarrow Y$. If $f \times i_{Z}: X \times Z \rightarrow Y \times Z$ is regular closed for all spaces $Z$, then $f$ is countably absolutely closed.

Proof. Since $f \times i_{Z}$ is regular closed for all spaces $Z, f$ is regular closed. Hence if $f$ is not countably absolutely closed then by Lemma 2 there is some $y_{0} \in Y$ for which $f^{-1}\left(y_{0}\right)$ is not c.f.f.r. So, let $\mathcal{U}$ be a free maximal countable open filter with $f^{-1}\left(y_{0}\right) \cap \bar{U} \neq \varnothing$ for all $U \in \mathcal{U}$. If $\left\{U_{i}\right\}$ is a base for $\mathcal{U}$ we may assume that $U_{n+1} \subseteq U_{n}$ for all $n$. Let $p$ be an additional point with basic neighborhoods $U_{i} \cup\{p\}$. Then if $Z=X \cup\{p\}, Z-X$ is first countable and $Z$ is Hausdorff, so consider $f \times i_{Z}: X \times Z \rightarrow Y \times Z$. Since $\{p\}=$ $\mathrm{\cap Cl}_{Z} U_{n}$ we may assume $U_{n-1}-\mathrm{Cl}_{Z} U_{n} \neq \varnothing$ for all $n$. Let $V_{1}=U_{1} \times\left(Z-\mathrm{Cl}_{Z} U_{1}\right), \quad V_{2}=U_{2} \times\left(U_{1}-\mathrm{Cl}_{Z} U_{2}\right), \cdots$,

$$
V_{n}=U_{n} \times\left(U_{n-1}-\mathrm{Cl}_{Z} U_{n}\right)
$$

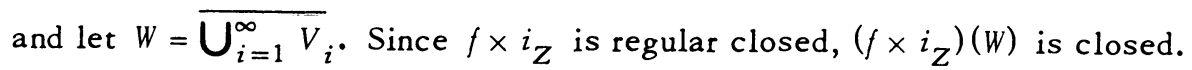
We claim that if $z \neq p,\left(y_{0}, z\right) \in\left(f \times i_{Z}\right)(W)$. Since $z \neq p$, there is a first $k$ for which $z \notin \bar{U}_{k}$, hence $z \in \overline{U_{k-1}}-\bar{U}_{k} \subseteq \overline{U_{k-1}-\bar{U}_{k}}$. Now, $f^{-1}\left(y_{0}\right) \cap$ $\bar{U}_{j} \neq \varnothing$ for all $j$, and $f$ is regular closed so that $y_{0} \in f\left(\bar{U}_{j}\right)=\overline{f\left(U_{j}\right)}$ for all $j$. Therefore,

$$
\begin{aligned}
\left(y_{0}, z\right) & \in \overline{f\left(U_{k}\right)} \times \overline{U_{k-1}-\bar{U}_{k}}=\overline{f\left(U_{k}\right) \times\left(U_{k-1}-\bar{U}_{k}\right)} \\
& =\overline{\left(f \times i_{Z}\right)\left(V_{k}\right)} \subseteq \overline{\left(f \times i_{Z}\right)\left(U V_{i}\right)}=\left(f \times i_{z}\right)(W),
\end{aligned}
$$

since $f \times i_{Z}$ is regular closed. Thus, $\left\{y_{0}\right\} \times X \subseteq\left(f \times i_{Z}\right)(W)$ so that $\left\{y_{0}\right\} \times$ $Z \subseteq\left(f \times i_{Z}\right)(W)$. It follows that there is some $x_{0} \in f^{-1}\left(y_{0}\right)$ with $\left(x_{0}, p\right) \in W$.

Let $K$ be the first integer with $x_{0} \notin \overline{U_{K}}$ and consider the neighborhood $\left(X-\bar{U}_{K}\right) \times U_{K+1}$ of $\left(x_{0}, p\right)$. If $\left(\left(X-\bar{U}_{K}\right) \times U_{K+1}\right) \cap\left(U_{N+1} \times\left(U_{N}-\bar{U}_{N+1}\right)\right)$ $\neq \varnothing$, then if $N \leq K$ then $U_{K} \subseteq U_{N}$ so that $U_{K+1} \cap\left(U_{N}-\overline{U_{N+1}}\right)=\varnothing$, while if $K<N, U_{N} \subseteq U_{K}$ so $U_{N+1} \cap\left(X-\bar{U}_{K}\right)=\varnothing$. In either case the contradiction implies that $\left(\left(X-\bar{U}_{k}\right) \times U_{k+1}\right) \cap \bigcup V_{i}=\varnothing$ and hence $\left(x_{0}, p\right) \notin \bar{U} V_{i}=W$. This contradiction establishes the theorem.

In the proof of Theorem 2, if $X$ is first countable so is $Z$. Also, since a first countable subset which is c.f.f.r. is easily first-countable-and-Haus- 
dorff-closed (closed in every first countable Hausdorff space in which it is embedded [5]) we have from Theorem 2,

Corollary 2.1. If $f \times i_{Z}: X \times Z \rightarrow Y \times Z$ is regular closed for all first countable spaces and $X$ is first countable, then $f^{-1}(y)$ is first-countableand-Hausdorff-closed for all $y \in Y$ and $f$ is countably absolutely closed.

Lemma 3 (Stephenson [5, Theorem 5.7]). For each first countable Hausdorff space $X$, the space $Z$ of all countable open ultrafilters on $X$ is firstcountable-and-Hausdorff-closed and contains $X$ as a dense subset.

Notice that in this extension we can take the neighborhoods of a point $p \in Z$ to be $\{p\} \cup U_{i}$, where $U_{i}$ is a member of the base for $p$. Then, in the proof of Theorem 2 we could have taken $Z$ to be this first countable Hausdorff extension of $X$, with $p \in Z-X$.

Corollary 2.2. If $X$ is first countable and $f \times i_{Z}: X \times Z \rightarrow Y \times Z$ is regular closed for all first-countable-and-Hausdorff-closed spaces $Z$, then $f$ is countably absolutely closed.

The next lemma is proved by using Viglino's ultrafilter characterization of absolutely closed maps [6, Theorem 1.2] (or Lemma 1 for the countable case) and imitating the proof of Lemma 2 of [2, p. 101]. Theorem 3 and its corollary then follow from the lemma and Theorem 1, and the lemma and Lemma 2 respectively.

Lemma 4. If $f_{\alpha}: X_{\alpha} \rightarrow Y_{\alpha}$ are (countably) absolutely closed for all $\alpha$ then so is $f: \Pi X_{\alpha} \rightarrow \Pi Y_{a},(f(x))_{\alpha}=f_{\alpha}\left(x_{a}\right)$.

Theorem 3. If $f: X \rightarrow Y$ is absolutely closed then $f \times i_{Z}: X \times Z \rightarrow$ $Y \times Z$ is regular closed for all spaces $Z$.

Corollary 3.1. If $f: X \rightarrow Y$ is countably absolutely closed and $Y$ is first countable, $f \times i_{Z}: X \times Z \rightarrow Y \times Z$ is regular closed for all first countable spaces $\mathrm{Z}$.

Corollary 3.2. If $X$ is first countable the following are equivalent:

(1) $\pi_{Z}: X \times Z \rightarrow Z$ is regular closed for all first countable spaces $Z$;

(2) $\pi_{Z}: X \times Z \rightarrow Z$ is regular closed for all first-countable-Hausdorffclosed spaces $Z$;

(3) $X$ is first-countable-and-Hausdorff-closed.

Proof. (1) $\Rightarrow$ (2) is obvious, (2) $\Rightarrow$ (3) follows from Corollary 2.2 by 
taking $Y$ to be a point, and $(3) \Rightarrow(1)$, follows from Corollary 3.1 again by letting $Y$ be a single point.

Added in proof. The following is proved by using Theorem 1 and applying the methods of the proof of Theorem 7 of [4].

Theorem. If $f \times i_{Z}: X \times Z \rightarrow Y \times Z$ is regular closed for all spaces $Z$, then $f$ is absolutely closed.

Question. If $f \times i_{Z}: X \times Z \rightarrow Y \times Z$ is regular closed for all $H$-closed spaces $Z$, is $f$ necessarily absolutely closed?

\section{BIBLIOGRAPHY}

1. A. Blaszczyk and J. Mioduszewski, On factorization of maps through $\tau X$, Colloq. Math. 23 (1971), 45-52. MR 46 \#4461.

2. N. Bourbaki, Eléments de mathématique. I: Les structures fondamentales de l'analyse. Livre III: Topologie générale, Actualités Sci. Indust., nos. 1029, 1045, 1084, 1142, 1143, Hermann, Paris, 1947, 1948, 1949; English transl., Hermann, Paris; Addison-Wesley, Reading, Mass., 1966. MR 34 \#5044b.

3. R. F. Dickman, Jr., Regular closed maps, Proc. Amer. Math. Soc. 39 (1973), 414-416. MR 47 \#4203.

4. C. T. Scarborough, Closed graphs and closed projections, Proc. Amer. Math. Soc. 20 (1969), 465-470. MR 40 \#3514.

5. R. Stephenson, Minimal first countable topologies, Trans. Amer. Math. Soc. 138 (1969), 115-127. MR 38 \#6537.

6. G. Viglino, Extensions of functions and spaces, Trans. Amer. Math. Soc. 179 (1973), 61-69.

DEPARTMENT OF MATHEMATICS, UNIVERSITY OF TEXAS, AUSTIN, TEXAS 78712

Current address: Department of Mathematics, University of Missouri, Columbia, Missouri 65201 